\title{
Cognitive Effort Modulates Connectivity between Dorsal Anterior Cingulate Cortex and Task-Relevant Cortical Areas
}

\author{
Bart Aben, ${ }^{1,2}$ Cristian Buc Calderon, ${ }^{2}$ Eva Van den Bussche, ${ }^{1}$ and ${ }^{\circledR}$ Tom Verguts ${ }^{2}$ \\ ${ }^{1}$ Faculty of Psychology and Educational Sciences, Brain and Cognition, KU Leuven, 3000 Leuven, Belgium, and ${ }^{2}$ Department of Experimental \\ Psychology, Ghent University, 9000 Ghent, Belgium
}

Investment of cognitive effort is required in everyday life and has received ample attention in recent neurocognitive frameworks. The neural mechanism of effort investment is thought to be structured hierarchically, with dorsal anterior cingulate cortex (dACC) at the highest level, recruiting task-specific upstream areas. In the current fMRI study, we tested whether dACC is generally active when effort demand is high across tasks with different stimuli, and whether connectivity between dACC and task-specific areas is increased depending on the task requirements and effort level at hand. For that purpose, a perceptual detection task was administered that required male and female human participants to detect either a face or a house in a noisy image. Effort demand was manipulated by adding little (low effort) or much (high effort) noise to the images. Results showed a network of AACC, anterior insula (AI), and intraparietal sulcus (IPS) to be more active when effort demand was high, independent of the performed task (face or house detection). Importantly, effort demand modulated functional connectivity between AACC and face-responsive or house-responsive perceptual areas, depending on the task at hand. This shows that dACC, AI, and IPS constitute a general effort-responsive network and suggests that the neural implementation of cognitive effort involves dACC-initiated sensitization of task-relevant areas.

Key words: anterior cingulate cortex; cognitive effort; fMRI; functional connectivity; PPI

Significance Statement

Although cognitive effort is generally perceived as aversive, its investment is inevitable when navigating an increasingly complex society. In this study, we demonstrate how the human brain tailors the implementation of effort to the requirements of the task at hand. We show increased effort-related activity in a network of brain areas consisting of dorsal anterior cingulate cortex (dACC), anterior insula, and intraparietal sulcus, independent of task specifics. Crucially, we also show that effortinduced functional connectivity between dACC and task-relevant areas tracks specific task demands. These results demonstrate how brain regions specialized to solve a task may be energized by dACC when effort demand is high.

\section{Introduction}

Cognitive effort plays an important role in many aspects of daily life. For example, its investment can lead to feelings of cognitive fatigue (Boksem and Tops, 2008; Inzlicht et al., 2018; Müller and Apps, 2019); it is perceived as aversive and is therefore avoided if possible (Kool et al., 2010; Kurzban et al., 2013); and it is registered as a cost in decision-making (Botvinick et al., 2009; Shenhav et al., 2013; Apps et al., 2015). Cognitive effort is distinct from task difficulty. Whereas effort is a property of the actor (i.e.,

\footnotetext{
Received Dec. 12, 2019; revised Mar. 24, 2020; accepted Mar. 25, 2020.

Author contributions: B.A., C.B.C., E.V.d.B., and T.V. designed research; B.A. and C.B.C. performed research; B.A. analyzed data; B.A., C.B.C., E.V.d.B., and T.V. wrote the paper.

The authors declare no competing financial interests.

This work was supported by the Research Foundation Flanders, Belgium (FWO Vlaanderen; Grant FW0TM765 to B.A.; Grant 1207719N to (.B.C.; and Grant FWOAL685 to E.V.d.B. and T.V.) and by the Excellence of Science Program (Grant GOF3818N to C.B.C. and T.V).

Correspondence should be addressed to Bart Aben at bart.aben@kuleuven.be.

https://doi.org/10.1523/JNEUROSCI.2948-19.2020

Copyright $\odot 2020$ the authors
}

defined as the investment of cognitive resources), difficulty is a property of the task (Inzlicht et al., 2018). For example, a task can be difficult without the actor investing much effort into it. Yet, effort and difficulty are also closely related: difficult stimuli will typically require and evoke (at least up to some difficulty level) a higher effort investment.

Cognitive effort can be conceptualized as the amplification of cognitive activity to resolve a demanding cognitive task (Inzlicht et al., 2018). Research on effort-based decision-making has identified a network of brain regions that are active when a person engages in effortful behavior. This circuitry mainly involves dorsal anterior cingulate cortex (dACC), anterior insula (AI), lateral prefrontal cortex (PFC), and intraparietal sulcus (IPS; Dosenbach et al., 2008; Menon and Uddin, 2010; Shenhav et al., 2013). Although dACC and AI in particular are often jointly activated across various tasks (Medford and Critchley, 2010; Nelson et al., 2010; Engström et al., 2015), it is generally thought that dACC is more directly involved in effort allocation. Instead, AI may signal the saliency of events (Menon and Uddin, 2010; Shenhav et al., 2017) 
A
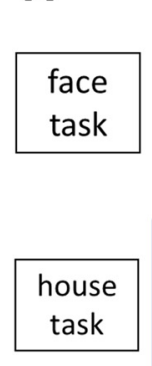

low effort
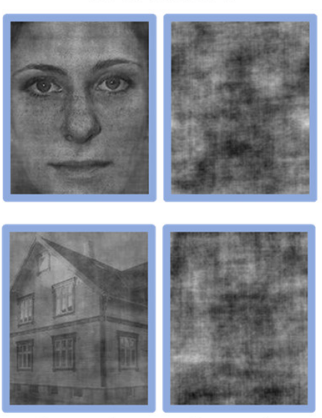

target

present

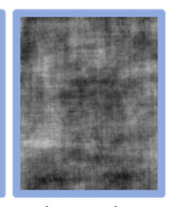

target

absent

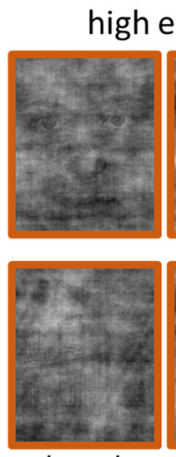

target

present
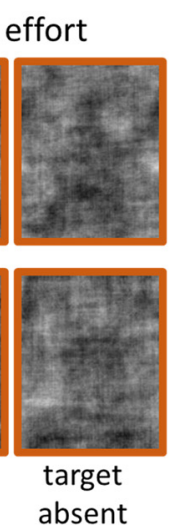

B

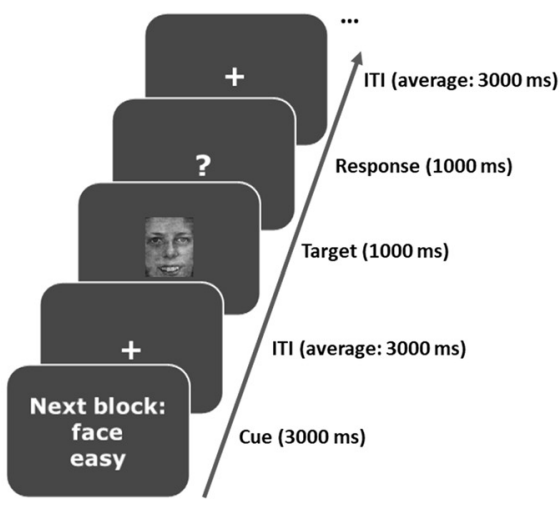

Figure 1. A, Examples of the face and house stimuli in the four conditions. The color coding is used for clarification only and was not used in the experiment. $\boldsymbol{B}$, Paradigm of the target detection tasks. Each block started with a cue signaling the task the subject had to perform: decide whether the image contained a face (target present) or not (target absent), or decide whether the image contained a house or not. The cue also indicated the effort level of the task (easy for low effort, difficult for high effort). The trial displayed here is an example of a targetpresent trial in the low-effort condition of the face detection task. ITI = intertrial interval.

or represent task-set maintenance (Dosenbach et al., 2006; Nelson et al., 2010). Therefore, recent frameworks have positioned dACC at the top of a hierarchy, exhibiting increased activity whenever effort demand is high, independent of the task that is performed (Holroyd and Yeung, 2012; Verguts et al., 2015). To solve a difficult task, it is believed that dACC, as part of a broader network of brain regions, recruits specialized upstream areas such as perceptual regions.

The activation of perceptual areas by top-down processes has been reported previously. For example, dACC is involved in topdown attentional modulation of task-relevant perceptual areas (Crottaz-Herbette and Menon, 2006; Danielmeier et al., 2011). The mere expectation of a face or house image can also be sufficient to evoke activity in fusiform face area (FFA) and parahippocampal place area (PPA), respectively (Summerfield et al., 2006; Esterman and Yantis, 2010). In the present fMRI study, we built on these findings to elucidate how effort investment is implemented in the brain and to investigate whether dACC indeed recruits specialized upstream areas when effort demand is high.

In two perceptual detection tasks with different stimulus types, subjects decided whether an image contained either a face or a house, or only noise. To disclose the top-down effects of effort investment on perceptual performance, we manipulated the effort required to solve the tasks by adding a little noise (loweffort trials) or much noise (high-effort trials) to the images. With much noise, the perceptual evidence contained by the image is low. On such difficult occasions, additional cognitive effort is required for the actor to reach an accurate decision. We hypothesized that this increased effort may serve as a top-down amplifier of the low signal-to-noise ratio, leading to an improvement in detection accuracy in noisy circumstances (Shenhav et al., 2013; Verguts et al., 2015). Specifically, we expected increased activation of effort-responsive areas such as AACC and AI, when effort demand is high, independent of the task that was performed. Importantly, because participants knew beforehand whether they had to detect a face or a house, we expected that dACC would increase its functional connectivity to FFA and PPA, respectively, when effort demand was high compared with low. This would show that dACC exerts its effects by modulating upstream areas, when effort investment is large. In addition to the a priori chosen analyses, we also explored whether errors and cognitive fatigue (i.e., time-on-task) affected behavioral and neural effort processing.

\section{Materials and Methods}

\section{Participants}

Thirty healthy Ghent University students gave written informed consent to participate (19 females, 11 males; mean age, 23.07 years; $\mathrm{SD}=3.48$; age range, $18-33$ years). The study was approved by the ethics committee of the Ghent University Hospital. All participants reported no history of psychiatric or neurologic disorder, were right handed, had normal vision, and were rewarded with 30 Euros in exchange for their participation.

\section{Stimuli}

Grayscale images of 18 faces and 18 houses were used (Fig. 1; Schiffer et al., 2014). Several measures were taken to nullify differences in surface properties between images. First, the images were equated in mean luminance level, contrast, and spatial frequency using the SHINE (Spectrum, Histology and Intensity Normalization and Equalization) toolbox (Willenbockel et al., 2010).

Next, for each trial, noise masks were created through image phase scrambling. Fast Fourier transforms were computed of one randomly selected face image and one randomly selected house image. The amplitude and phase matrices of these images were averaged across the two images. A random phase matrix was added to the averaged phase matrix, resulting in $100 \%$ phase scrambling. The final noise mask was obtained through inverse fast Fourier transformation of the averaged amplitude matrix and the averaged phase-plus-noise matrix.

The following two different trial types were created: target-present trials and target-absent trials. On target-present trials, a face or house image was blended with a noise mask. The blending depended on a threshold corresponding to a participant-specific and task-specific interpolation factor. These thresholds were obtained from a staircase procedure performed before the main task (see Staircase procedure). The thresholds could vary from 0 , meaning that the original face or house image was returned, to 1 , meaning that the noise mask was returned.

Each target-absent trial consisted of a superimposed face and a house image (i.e., they were blended with interpolation factor 0.5). Next, the superimposed image was $100 \%$ phase scrambled. This phase-scrambled image was in turn blended with the noise mask, with an interpolation factor that was the average of the face and house threshold obtained from the participant's staircase performance (see below). This procedure assured identical target-absent trials in all conditions.

\section{Experimental design and statistical analysis}

Target detection tasks. On each trial, participants were shown a noisy image displayed against a gray background at $6.2^{\circ} \times 8^{\circ}$ visual angle (Fig. $1 B)$. Two different target detection tasks were administered. In the face task, participants had to decide whether the noisy image contained a face (target present) or not (target absent); in the house task, they had to 
decide whether the image contained a house or not (Fig. 1B). Participants pressed a button either with the right index or middle finger for a target-present or target-absent trial. Response mapping was counterbalanced across participants. Each trial consisted of a $1000 \mathrm{~ms}$ image presentation, during which participants were not allowed to respond, followed by a $1000 \mathrm{~ms}$ response window during which a question mark was displayed, and responses were recorded. Trials were separated by a pseudoexponentially distributed intertrial interval ranging from 1000 to $7000 \mathrm{~ms}$ (average, $3000 \mathrm{~ms}$ ) during which a fixation cross was displayed.

Each task was performed at the following two effort levels: in the low-effort condition, the difference in target visibility between targetpresent and target-absent trials was large; in the high-effort condition, this difference was small (Fig. 1A). Specifically, the effort level of each block depended on the interpolation level of the target images. In loweffort blocks, the interpolation level of each target image was randomly drawn from a normal distribution centered at $\sim 0.30$ with $\mathrm{SD}=0.07$. This resulted in a clear difference between target and noise trials (Fig. $1 A$ ). In high-effort blocks, the interpolation level of target images was randomly drawn from a normal distribution centered around the threshold obtained through a staircase procedure (see Staircase procedure), with $\mathrm{SD}=0.07$. Hence, on high-effort blocks, the difference between target and noise images was much smaller (Fig. 1A).

Task (face vs house detection) and Effort (low vs high) were varied blockwise, resulting in the following four different block types, or conditions: face low-effort; face high-effort; house low-effort; and house high-effort (Fig. 1A). Each block contained 18 trials, half of which were target-present trials while the other half were targetabsent trials. Blocks were presented in five rounds, resulting in a total of 90 trials per condition. Each round contained a single presentation of all block types in random order.

Participants were made aware of the difference between low-effort and high-effort blocks through verbal instructions before the experiment started. They were also cued at the start of each block about which task to perform (i.e., detect face or detect house) and whether the block was a low-effort block (i.e., "easy") or a high-effort block (i.e., "difficult"; Fig. $1 B)$. Participants were instructed to stay focused on the center of the images to avoid eye movements. Accuracy feedback was provided at the end of each block.

Target detection tasks: $d^{\prime}$ analysis. For each participant and condition (face low-effort, face high-effort, house low-effort, and house higheffort), we computed the $d^{\prime}$ value to evaluate performance. $d^{\prime}$ is a measure of the difference between the mean sensory activity generated by a signal-plus-noise trial versus a noise-alone trial, expressed in $z$-scores. A $2 \times 2$ repeated-measures ANOVAs with the factors Task (face vs house) and Effort (low effort vs high effort) was performed on these $\mathrm{d}^{\prime}$ measures. Trials with no response were excluded from analysis (2.9\%).

To explore the effects of cognitive fatigue (i.e., time-on-task) on behavior, the behavioral data were split in early stages (i.e., first 180 trials) and late stages (i.e., last 180 trials). The ANOVA on the $\mathrm{d}^{\prime}$ scores was repeated with the additional factor Stage (early vs late).

Subjective rating task. To ensure that the high-effort conditions of the target detection tasks were indeed perceived as more difficult than the low-effort conditions and that participants indeed invested more effort into it, a separate sample of 32 first-year psychology students was recruited (27 females, 5 males; mean age, 18.72 years; SD, 1.30; age range, $18-23$ years). All participants gave written informed consent and were rewarded with one course credit. They were administered the same target-detection tasks described above, outside the scanner, and with the addition of two questions after each block of trials. One question probed task difficulty: "How difficult did you find the previous block?" The other probed invested effort: "How much effort did it take you to complete the previous block?" Participants rated difficulty and effort investment on a horizontal line ranging from 0 ("not difficult at all" or "no effort at all") to 100 ("extremely difficult" or "extreme effort"). The questions were presented in random order after each block.

Subjective rating task analysis. The ratings of difficulty and effort investment were averaged per participant and condition. These ratings were subjected to a repeated-measures ANOVA with the factors Task and Effort.
Staircase procedure. Before the target detection task, participants performed a linear adaptive one-up/two-down staircase outside the scanner to determine the interpolation factor for each participant for the higheffort blocks. This was done in two separate blocks for face and house images. The trial procedure was similar to the main task, except for the inclusion of feedback on accuracy ("correct" and "false") and response time ("too slow" when the response deadline of $1000 \mathrm{~ms}$ was exceeded). Participants performed two blocks of 72 trials separated by a $10 \mathrm{~s}$ pause. Based on pilot data, the initial interpolation level was set at 0.65 for each block. The interpolation level dropped with one step after every incorrect response and increased with one step after two consecutive correct responses. For the first four reversals, the step size was set at 0.02 to accelerate progressing through the staircase in the beginning. From the fifth reversal, the step size was set to 0.01 . The average interpolation level on the last six reversals was taken as the participant's interpolation factor. The mean interpolation level was 0.80 (SD, 0.02; range, 0.71-0.83) for the face task and 0.77 (SD, 0.03; range, 0.70-0.82) for the house task. Block order was counterbalanced between participants. Response configuration was kept identical between the staircase task and the target detection task.

fMRI acquisition. Images were collected by means of a 3 tesla Magnetom Trio MRI scanner system (Siemens Medical Systems), with a 64-channel radiofrequency head coil. Participants perceived stimuli projected onto a screen at the extremity of the magnet bore through a mirror mounted on the head coil. Stimulus presentation was controlled by PsychoPy version 1.85.4 (Peirce, 2007). First, a high-resolution T1weighted structural scan (MP-RAGE) was conducted (176 slices; $1 \mathrm{~mm}$ slice thickness; TR, $2250 \mathrm{~ms}$; TE, $4.18 \mathrm{~ms}$; flip angle, $9^{\circ}$ ). This was followed by two functional runs using a gradient echo echoplanar pulse sequence. Functional images consisted of 38 axial slices ( $3.5 \mathrm{~mm}$ thick; 1 mm skip, 229 field of view), with $\mathrm{TR}=2 \mathrm{~s}, \mathrm{TE}=29 \mathrm{~ms}$, flip angle $=90^{\circ}$, and $3.5 \times 3.5 \times 3.5 \mathrm{~mm}$ in-plane resolution.

fMRI data analysis: data preprocessing. The fMRI data were preprocessed and analyzed using SPM12 (http://www.fil.ion.ucl.ac.uk/spm/ software/spm12). Functional data were corrected for differences in acquisition times between slices for each whole-brain volume, realigned within runs, and coregistered with each participant's anatomic scan. The functional data were then segmented and spatially normalized to standard MNI space (2 mm isotropic voxels). Normalized data were spatially smoothed (6 mm full-width at half-maximum) using a Gaussian kernel filter. Six motion parameters were estimated using the Artifact Detection Tool software package (ART; https://www.nitrc.org/projects/artifact_ detect). These parameters were used to check for outlier scans, which were identified in the temporal differences series by assessing betweenscan differences using a $z$-threshold of $5.0 \mathrm{~mm}$ and a scan-to-scan movement threshold of $0.9 \mathrm{~mm}$. The motion parameters and outlier regressors identified with ART were included in all first-level statistical analyses as nuisance regressors.

fMRI data analysis: Target detection task. Using a general linear model (GLM), blood oxygenation level-dependent (BOLD) responses for all participants were modeled at each voxel. Initially, a hybrid design modeling both transient trial responses and sustained block responses was explored in one design (Visscher et al., 2003; Petersen and Dubis, 2012). However, the block regressors had to be dropped because of high collinearity. The remaining design included four trial types, resulting from the crossing of the factors Task (face vs house) and Effort (low effort vs high effort). Trial regressors were convolved with a canonical hemodynamic response function (HRF), including time and dispersive derivatives. The event length was set at $0 \mathrm{~s}$. The six motion parameters and outlier regressors identified with ART were included as nuisance regressors. A high-pass filter of $0.008 \mathrm{~Hz}$ was applied, and temporal autocorrelations were accounted for using the default first-order autoregressive $[\mathrm{AR}(1)]$ model.

We first checked for task-specific effects by computing contrasts between the face and house tasks for low-effort and high-effort conditions. In a similar vein, contrasts were computed between low-effort and high-effort conditions for face and house tasks to identify effort effects. Individual contrast maps were subjected to second-level random-effects models. Significance was tested through one-sample $t$ tests. Since FFA 
activity is generally more robust in right hemisphere than in left hemisphere in right handers (Kanwisher et al., 1997; Willems et al., 2010), we also applied a small volume correction in case bilateral activity was not found in whole-brain analyses. Small volume correction was performed with $10 \mathrm{~mm}$ bilateral spherical ROIs centered at the mean peak voxels obtained from the localizer. To determine which regions were responsive to high-effort demand across tasks, a conjunction analysis was performed on the effort activation maps of the face and house conditions. The conjunction null hypothesis was assessed (Nichols et al., 2005), meaning that only regions significant in both contrasts survived.

To explore whether results were affected by response accuracy, we also created a new GLM where we added parametric modulators for accuracy $(0=$ correct, $1=$ error $)$. Because few errors were anticipated for the low-effort condition, and, to not overparameterize the GLM, these modulators were only applied to the high-effort conditions (FaceHE, HouseHE). Further analyses were identical to the original GLM, with additional one-sided $t$ tests on the regressors for the parametric modulation.

Results are reported at an uncorrected voxel-based threshold of $p<0.001$ and were cluster corrected to control the FWE at $p=0.05$. For the visualization of results, statistical maps were projected onto $2 \mathrm{D}$ slices using MRIcroGL (https://www.nitrc.org/projects/mricrogl) and on cortical surfaces with the use of Surf Ice (https://www.nitrc.org/projects/ surfice).

fMRI data analysis: Functional connectivity. A generalized psychophysiological interaction (gPPI) analysis was conducted to assess whether connectivity between effort-responsive regions (dACC) and lower-level sensory cortices increased in a task-dependent way. This analysis was conducted on unsmoothed volumes with the CONN toolbox (http://www.conn-toolbox.org; Whitfield-Gabrieli and NietoCastanon, 2012). To remove non-neural sources from the neural signal, an anatomic component-based noise correction (aCompCor) strategy was used (Behzadi et al., 2007). The six realignment parameters were regressed out, and the BOLD signals from individual white matter and CSF masks were used to remove noise components. Next, the gPPI model was estimated according to the following:

$$
\begin{aligned}
& y=\beta_{0}+\beta_{1} S+\beta_{2} \text { FaceLE }+\beta_{3} \text { FaceHE }+\beta_{4} \text { HouseLE }+\beta_{5} \text { HouseHE } \\
& +\beta_{6} \text { FaceLE } \times \mathrm{S}+\beta_{7} \text { FaceHE } \times \mathrm{S}+\beta_{8} \text { HouseLE } \times \mathrm{S}+\beta_{9} \text { HouseHE } \times \mathrm{S},
\end{aligned}
$$

where $S$ is the averaged time series of the seed region; FaceLE, FaceHE, HouseLE, and HouseHE are the psychological (block) regressors representing the four conditions; and FaceLE $\times S$, FaceHE $\times S$, HouseLE $\times S$, and HouseHE $\times S$ are the psychophysiological interactions between the psychological regressors and the averaged time series of the seed.

Whereas the activity analyses were performed on trial level, the connectivity analysis used the blocked structure of the design. This way, both analyses leveraged specific assets of the design. In the activity analyses, the task events of noninterest were excluded, resulting in a cleaner BOLD signal. In the connectivity analysis, block regressors increased power by stretching events across longer time periods (GonzalezCastillo et al., 2015). Also, with block regressors, how exactly the seed and task time series are deconvolved is of little influence, since blocked regressors will saturate to look like a block independent of the deconvolution method (O'Reilly et al., 2012; Di and Biswal, 2017). In the current study, the psychological regressors were convolved with the hemodynamic response function and the interactions were modeled on the raw BOLD-level signal.

The gPPI analysis was first conducted on whole-brain level, assessing seed-to-voxel connectivity. The individual $\beta$ values corresponding to the interaction terms ( $\beta_{6}$ to $\beta_{9}$ ) were subjected to a second-level random-effects analysis, where we contrasted low-effort and high-effort blocks within each task (i.e., FaceHE $>$ FaceLE; HouseHE $>$ HouseLE) and assessed the difference between these two contrasts (i.e., [FaceHE > FaceLE] vs [HouseHE $>$ HouseLE]).

The whole-brain analysis was followed-up by an ROI-to-ROI analysis, where we estimated the interaction terms $\left(\beta_{6}\right.$ to $\left.\beta_{9}\right)$ using the averaged time series of individual FFA and PPA target ROIs (see Localizer). The interaction terms were first subjected to multivariate $F$ tests to jointly evaluate whether the connectivity between the seed and any of the target ROIs showed any significant effect of interest. This was followed by false discovery rate (FDR)-corrected individual $t$ tests between the seed and each of the target ROIs.

Two additional connectivity analyses were conducted. First, the ROIto-ROI analysis was replicated after extending the original gPPI analysis with parametric modulators for accuracy $(0=$ correct, $1=$ error $)$. Second, to explore whether connectivity was affected by cognitive fatigue (i.e., time-on-task), the ROI-to-ROI analysis was also replicated after extending the original gPPI with parametric modulators for Stage (i.e., $0=$ early, 1 = late; see also Target detection tasks: $\mathrm{d}^{\prime}$ analysis). Parametric modulators were multiplied by the averaged time series of the seed to create the relevant psychophysiological interaction regressors. Modulations were applied only to the high-effort conditions (FaceHE, HouseHE) to not overparameterize the model, because few errors were anticipated in the low-effort conditions, and because the effects of cognitive fatigue were mainly expected in the high-effort conditions. Both additional connectivity analyses were conducted only through ROI-to-ROI analyses, as these are the most powerful analyses to explore effects of accuracy and cognitive fatigue on connectivity. Further analyses were identical to the original ROI-to-ROI analysis, with additional $F$ and $t$ tests on the regressors for the respective parametric modulations.

fMRI data analysis: Localizer task. A functional localizer task was administered after the main experiment to identify face-selective and house-selective areas (i.e., FFA and PPA, respectively). Participants performed a 1-back task where they had to press a button with the right index finger for any immediate repetition of an image. The same face and house images as in the target detection task were presented blockwise in eight 18-trial blocks of each type (16 blocks in total). No noise was added to the images. Face and house blocks alternated. Which block was presented first was counterbalanced across participants. Image presentation within blocks was random. Images were displayed for $1500 \mathrm{~ms}$ and separated by a $500 \mathrm{~ms}$ fixation cross. Mean accuracy on immediate repetitions was $88.8 \%$ on face trials and $90.4 \%$ on house trials $\left(t_{(29)}=\right.$ $-0.76, p=0.46$ ).

Blocks of face and house images were modeled by boxcar functions convolved with the canonical HRF and its time derivative. The lengths of the boxcars corresponded with the block lengths. To obtain individual ROIs, the following procedure was implemented. First, a GLM was created with regressors for face and house blocks. Individual contrast maps were computed comparing face versus house and vice versa, using an uncorrected voxel-based threshold of $p<0.001$ and cluster correction to control the FWE at $p=0.05$. Second, FDR-corrected activation maps were obtained from the Neurosynth database (http://www.neurosynth. org; Yarkoni et al., 2011) using the search terms "fusiform" $(n=972)$, "fusiform face" ( $n=143)$, "fusiform gyrus" $(n=91)$, and "fusiform gyri" $(n=582)$ for FFA; and "parahippocampal" $(n=602)$, "parahippocampal cortex" $(n=76)$, and "parahippocampal gyrus" $(n=327)$ for PPA. These maps were binarized and combined into two single-activation maps (one for bilateral FFA, one for bilateral PPA). Finally, the intersections between each Neurosynth activation map and the corresponding individual contrast map from the localizer task were computed. Six millimeter spheres were created, centered on the peak voxel coordinates within the remaining left and right clusters of the two intersection contrasts (i.e., face vs house and house vs face). This resulted in four ROIs for each participant: left FFA, right FFA, left PPA, and right PPA (Fig. 2).

\section{Eye movement data analysis}

Eye movements of 24 participants were recorded with a long-range optics infrared eye tracker (EyeTrac 6, Applied Science Laboratories). Eye data of six participants were not recorded due to technical difficulties. Data were collected at a sampling rate of $120 \mathrm{~Hz}$. Fixation epochs were computed to determine whether participants refrained from eye movements during trials. A fixation epoch started when six consecutive samples fell within an SD of 0.5 visual degrees. It ended when three consecutive samples fell outside of a $1.5^{\circ}$ ellipse around the original fixation 


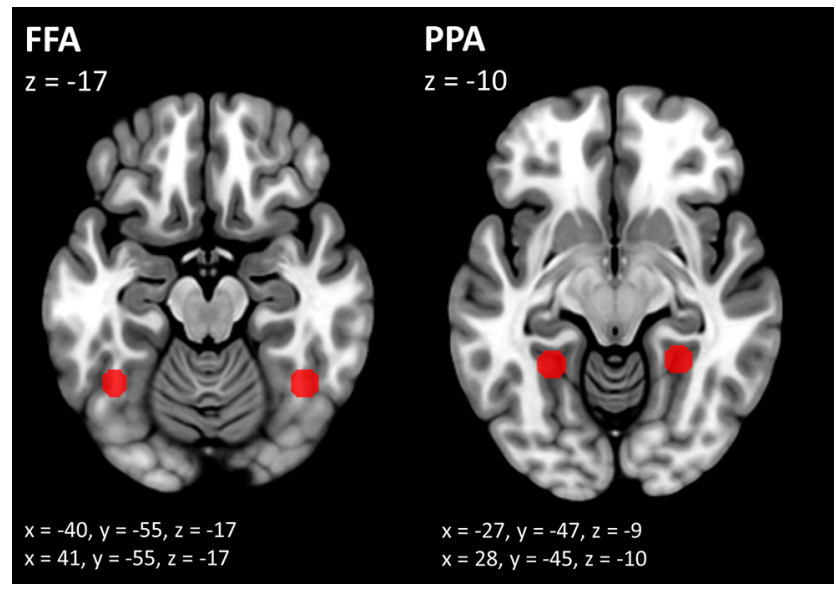

Figure 2. Location of FFA and PPA ROIs obtained from the functional localizer, averaged across participants.

point. Trials that were completely within one fixation epoch were marked as fixation trials.

The percentage of fixation trials per participant and condition was computed. These percentages were subjected to a $2 \times 2$ repeated-measures ANOVA with the factors Task (face vs house) and Effort (low effort vs high effort) to check for differences in eye movements between conditions.

\section{Results}

\section{Behavior}

Target detection task: $d^{\prime}$

Effects are displayed in Figure 3A. An ANOVA was conducted on d' scores with the factors Task (face vs house) and Effort (low vs high). A main effect of Task was found, with higher $\mathrm{d}^{\prime}$ scores on the face task (mean, 2.46) than on the house task (mean, 2.15; $\left.F_{(1,29)}=23.21 ; p<0.001 ; \eta^{2}=0.100\right)$. A main effect of Effort was also present, with higher $\mathrm{d}^{\prime}$ scores in the low-effort condition (mean, 3.72) than in the high-effort condition (mean, 0.89), $F_{(1,29)}=846.56, p<0.001, \eta^{2}=0.901$. An interaction between Task and Effort was found $\left(F_{(1,29)}=5.06 ; p=0.032 ; \eta^{2}=0.032\right)$, indicating that the difference between low-effort and high-effort conditions was larger on the face task (mean, 3.96 vs 0.97 ) than on the house task (mean, 3.50 vs 0.82 ). Importantly, the $\mathrm{d}^{\prime}$ scores of all four conditions differed from zero ( $p$ values $<0.001)$, including the high-effort face condition $\left(t_{(29)}=11.72 ; p<0.001\right.$; $d=2.140)$ and the high-effort house condition $\left(t_{(29)}=13.68\right.$; $p<0.001 ; d=2.498)$. This indicates that participants were engaged in the task and able to discriminate between target-present and target-absent trials, also on the high-effort blocks.

To explore the effects of cognitive fatigue (i.e., time-on-task), the analysis of the $\mathrm{d}^{\prime}$ scores was repeated after splitting the data in half. The original ANOVA was extended with the factor Stage (early vs late). Because the split halved the number of trials in each cell, some participants had a miss rate or false alarm rate of 0 in some conditions. This was corrected using log-linear transformations following recommendations of Hautus (1995). The only significant effect that involved Stage was the three-way interaction among Task, Block, and Stage $\left(F_{(1,29)}=6.65 ; p=\right.$ $\left.0.015 ; \eta^{2}=0.011\right)$. To decompose this interaction, post hoc paired-samples $t$ tests were conducted comparing the early to the late stage in each condition. This revealed that performance deteriorated over time in the low-effort face condition (mean $\mathrm{d}^{\prime}$ in early stage $=3.60 ;$ mean $\mathrm{d}^{\prime}$ in late stage $=3.42 ; t_{(29)}=2.27$; $p=0.031 ; d=0.451)$. No effects of Stage were found in the other conditions ( $p$ values $>0.17$. Note that because many subjects had nearly perfect scores in the low-effort conditions, a few slips in the second stage of the task may already have led to a significant difference with the early stage, offering a possible explanation for the observed effect.

\section{Subjective rating task}

The subjective ratings are displayed in Figure 3, $B$ and $C$. A main effect of Effort on difficulty rating was found, with higher ratings in the high-effort condition (mean, 71.2) than in the low-effort condition (mean, 23.2; $F_{(1,31)}=157.62 ; p<0.001 ; \eta^{2}=0.710$; Fig. $3 B$ ). No other effects on difficulty rating were found ( $p$ values $>0.43$. A main effect of Effort on effort investment rating was also found, with higher ratings in the high-effort condition (mean, 59.0) than in the low-effort condition (mean, 28.7; $F_{(1,31)}=$ 58.02; $p<0.001 ; \eta^{2}=0.422$; Fig. $3 C$ ). No other effects on effort investment rating reached significance ( $p$ values $>0.37$ ).

\section{fMRI results}

Task-specific effects

Table 1 displays task-specific activation patterns in the low-effort and high-effort conditions. As anticipated, areas related to the processing of faces, such as right FFA and superior temporal sulcus showed increased activation in the low-effort condition of the face task (vs the low-effort house condition). After small volume correction, increased activity was also found in left FFA for this contrast. On the low-effort house task (vs the low-effort face task), areas responsive to house images, such as bilateral PPA, showed increased activation. In the high-effort face task (vs the high-effort house condition), activation in bilateral FFA was found after small volume correction. In the high-effort house condition (vs the high-effort face condition), bilateral PPA was activated. This shows that FFA and PPA were involved in the low-effort and high-effort condition, even despite the minor perceptual evidence in the high-effort condition.

\section{Effort-specific effects}

To investigate brain areas that are involved in effort investment in a task-general way, we first mapped areas responsive to high-effort demands in both tasks. In the face task, this revealed stronger activation when effort was high (vs low) in a network constituting dACC (extending into pre-SMA), bilateral AI, bilateral IPS, bilateral middle occipital gyrus (MOG), right inferior frontal gyrus (IFG), left cerebellum, and right dorsolateral PFC (dlPFC; Table 2, Fig. 4A). In the house task, we found effort-induced activation in right dACC (extending into pre-SMA), bilateral AI, right IPS, and bilateral MOG (Table 2, Fig. 4B). Next, a conjunction analysis was performed on the two contrasts. This revealed that voxels in right dACC, bilateral AI, and right IPS were conjointly activated (Table 2, Fig. 4C), showing that activation in these areas scaled with effort, independent of the task at hand.

Finally, no significant activation was found for the interaction between Task and Effort, indicating that, although activation of task-sensitive areas appeared to be stronger in the low-effort (vs high-effort) conditions, these differences did not survive statistical thresholding. Assuming stronger bottom-up activation of task-specific areas on perceptually strong low-effort trials (vs ambiguous high-effort trials), an interaction may have been expected. An opposite interaction would also have been plausible, if high-effort trials (vs low-effort trials) had elicited stronger top-down activation of task-specific areas. Note that because the two mechanisms operate in opposite directions, they may have countered each other. 
A

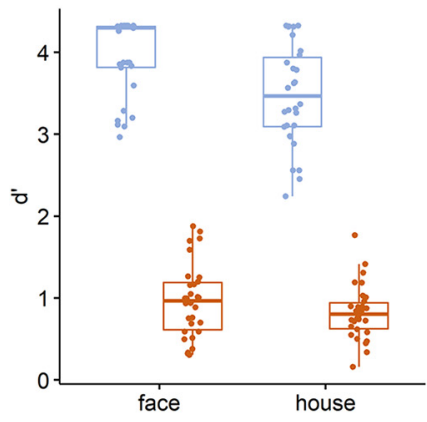

B

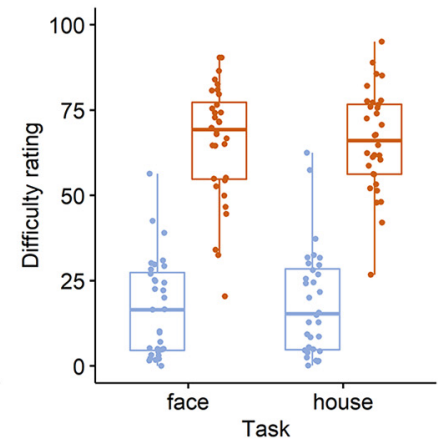

C

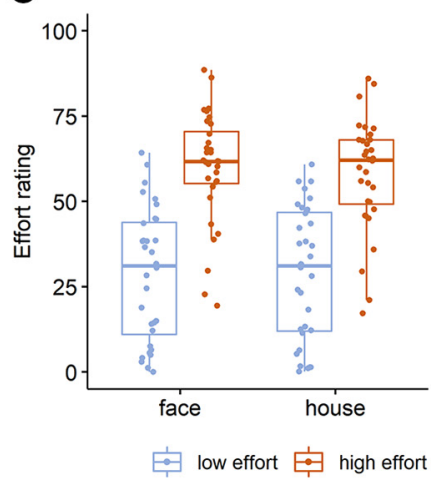

Figure 3. $A, d^{\prime}$ scores on the target detection task. $\boldsymbol{B}$, Subjective difficulty ratings. C, Subjective effort investment ratings.

Table 1. Summary of the task-specific activation clusters

\begin{tabular}{|c|c|c|c|c|c|c|c|}
\hline \multirow[b]{3}{*}{ Region } & \multirow[b]{3}{*}{ Side } & \multirow{2}{*}{\multicolumn{2}{|c|}{$\begin{array}{l}\text { Cluster-level } \\
\text { statistics }\end{array}$}} & \multicolumn{4}{|c|}{ Peak-level statistics } \\
\hline & & & & \multirow[b]{2}{*}{$t$ value } & \multicolumn{3}{|c|}{ MNI coordinates } \\
\hline & & Size & $p$ (FWE) & & $x$ & $y$ & $z$ \\
\hline \multicolumn{8}{|l|}{ Low effort: face $>$ house } \\
\hline Superior temporal sulcus & $\mathrm{R}$ & 427 & $<0.001$ & 5.77 & 58 & -58 & 10 \\
\hline Fusiform face area & $\mathrm{R}$ & 128 & 0.018 & 5.24 & 42 & -60 & -20 \\
\hline Precuneus & $\mathrm{R}$ & 279 & $<0.001$ & 4.94 & 2 & -50 & 42 \\
\hline Fusiform face area* & $\mathrm{L}$ & 21 & 0.013 & 4.69 & -42 & -62 & -18 \\
\hline \multicolumn{8}{|l|}{ Low effort: house $>$ face } \\
\hline Parahippocampal place area & $\mathrm{R}$ & 457 & $<0.001$ & 8.50 & 24 & -42 & -10 \\
\hline Parahippocampal place area & $\mathrm{L}$ & 288 & $<0.001$ & 6.72 & -24 & -46 & -10 \\
\hline Occipito-temporal cortex & $\mathrm{R}$ & 495 & $<0.001$ & 6.21 & 38 & -78 & 14 \\
\hline Occipito-temporal cortex & $\mathrm{L}$ & 303 & $<0.001$ & 5.43 & -32 & -88 & 12 \\
\hline \multicolumn{8}{|l|}{ High effort: face $>$ house } \\
\hline Fusiform face area* & $\mathrm{R}$ & 74 & 0.002 & 5.45 & 40 & -46 & -18 \\
\hline Fusiform face area* & L & 55 & 0.003 & 5.20 & -40 & -46 & -18 \\
\hline \multicolumn{8}{|l|}{ High effort: house $>$ face } \\
\hline Parahippocampal place area & $\mathrm{R}$ & 256 & 0.001 & 7.09 & 34 & -40 & -8 \\
\hline Parahippocampal place area & $\mathrm{L}$ & 213 & $<0.001$ & 6.82 & -26 & -44 & -10 \\
\hline
\end{tabular}

L, Left; $\mathrm{R}$, right.

*After small volume correction using a sphere with $10 \mathrm{~mm}$ radius centered at the peak voxel coordinates obtained from the localizer (Fig. 2).

To explore whether activity profiles were affected by response accuracy, we created a new GLM with additional parametric modulators for versus incorrect trials. These modulators show which voxels responded differently on incorrect (vs correct) trials. No modulation of brain activity by accuracy was found, suggesting that brain responses did not differ between correct and incorrect trials.

\section{Functional connectivity}

For the gPPI analysis, the dACC cluster that showed activation in the high-effort versus low-effort conjunction analysis was used as a seed. When comparing high-effort to low-effort demand in the face task, increased connectivity was found between $\mathrm{dACC}$ and multiple regions, including bilateral FFA (Table 3, Fig. 5A). The same contrast on the house task showed activation in several regions including right PPA and right FFA (Fig. 5B). An interaction contrast comparing effort effects between the face and house task (i.e., [FaceHE $>$ FaceLE] vs [HouseHE $>$ HouseLE]) revealed no significant activation.

To further investigate these connectivity patterns, we conducted an ROI-to-ROI gPPI analysis with the same dACC seed and the participant-specific (left and right) PPA and FFA ROIs obtained through the localizer task. This showed increased connectivity between dACC and the set of target ROIs (left PPA, right PPA, left FFA, and right FFA) when effort demands were high (vs low) in the face task $\left(F_{(4,26)}=6.84 ; p<0.001\right)$. Specifically, increased connectivity was found from dACC to left FFA $\left(\beta=0.17 ; t_{(29)}=4.54 ; p<0.001\right)$ and right FFA $(\beta=0.16$; $t_{(29)}=4.63$; $p<0.001$; Fig. $6 A$ ). When effort demands were high (vs low) in the house task, increased connectivity between dACC and the set of target ROIs was also observed $\left(F_{(4,26)}=4.45\right.$; $p=0.007)$. Specifically, this connectivity was from dACC to left PPA $\left(\beta=0.11 ; t_{(29)}=2.78 ; p=0.015\right)$, right PPA $(\beta=0.13$; $\left.t_{(29)}=3.65 ; p=0.004\right)$, and right FFA $\left(\beta=0.11 ; t_{(29)}=2.70\right.$; $p=0.015$; Fig. $6 B$ ). Crucially, the interaction contrast comparing effort effects between the face and house task (i.e., [FaceHE > FaceLE] vs [HouseHE $>$ HouseLE]) showed directly that connectivity patterns differentiated between tasks $\left(F_{(4,26)}=7.44\right.$; $p<0.001$; Fig. $6 C$ ). Effort-increased connectivity from dACC to left FFA was larger on the face task $\left(\beta=0.16 ; t_{(29)}=3.65\right.$; $p=0.004)$, while effort-increased connectivity from dACC to left PPA $\left(\beta=-0.12 ; t_{(29)}=-2.29 ; p=0.040\right)$ and right PPA $(\beta=$ $\left.-0.12 ; t_{(29)}=-2.83 ; p=0.017\right)$ was larger on the house task.

To explore whether ROI-to-ROI connectivity profiles differed between correct and incorrect trials, we created a new gPPI model with additional parametric modulators for correct versus incorrect responses. Modulation was only applied to the higheffort conditions (FaceHE, HouseHE). These modulators show in which ROIs the task-specific connectivity with dACC was different on incorrect (vs correct) trials. No association between connectivity and response accuracy was found in any of the ROIs in the high-effort face condition $\left(F_{(4,26)}=0.42 ; p=0.795\right)$ or in the high-effort house condition $\left(F_{(4,26)}=0.83 ; p=0.519\right)$. Thus, connectivity between dACC and target ROIs was not affected by errors.

Finally, an exploratory analysis was also conducted to test for effects of cognitive fatigue (i.e., time-on-task) on brain connectivity. An ROI-to-ROI analysis was performed after extending the original model with parametric modulators for Stage (early vs late) applied to the high-effort conditions (FaceHE, HouseHE). These modulators show ROIs in which the task-specific connectivity with dACC was different during the late (vs the early) stage of the task. No association was found between connectivity and Stage in any of the ROIs in the high-effort face condition $\left(F_{(4,26)}=0.80\right.$; $p=0.538)$ or in the high-effort house condition $\left(F_{(4,26)}=1.39\right.$; $p=0.264)$. Thus, connectivity between dACC and target ROIs was not affected by cognitive fatigue (i.e., time-on-task). 
Table 2. Summary of the activation clusters on the high-effort versus low-effort contrasts

\begin{tabular}{|c|c|c|c|c|c|c|c|}
\hline \multirow[b]{3}{*}{ Region } & \multirow[b]{3}{*}{ Side } & \multirow{2}{*}{\multicolumn{2}{|c|}{$\begin{array}{l}\text { Cluster-level } \\
\text { statistics }\end{array}$}} & \multicolumn{4}{|c|}{ Peak-level statistics } \\
\hline & & & & \multirow[b]{2}{*}{$t$ value } & \multicolumn{3}{|c|}{ MNI coordinates } \\
\hline & & Size & $p($ FWE $)$ & & $x$ & $y$ & $Z$ \\
\hline \multicolumn{8}{|l|}{ Face task: high effort $>$ low effort } \\
\hline Anterior insula & $\mathrm{R}$ & 546 & $<0.001$ & 8.63 & 32 & 20 & -4 \\
\hline Dorsal anterior cingulate cortex & $\mathrm{R}$ & 919 & $<0.001$ & 8.07 & 8 & 24 & 40 \\
\hline Anterior insula & $\mathrm{L}$ & 406 & $<0.001$ & 7.62 & -34 & 20 & -2 \\
\hline Intraparietal sulcus (extending into middle occipital gyrus) & $\mathrm{R}$ & 2084 & $<0.001$ & 7.56 & 38 & -78 & 12 \\
\hline Middle occipital gyrus & $\mathrm{L}$ & 1085 & $<0.001$ & 6.93 & -30 & -82 & 14 \\
\hline Intraparietal sulcus & $\mathrm{L}$ & 209 & 0.002 & 6.12 & -28 & -50 & 50 \\
\hline Inferior frontal gyrus & $\mathrm{R}$ & 361 & $<0.001$ & 5.89 & 48 & 10 & 22 \\
\hline Cerebellum & $\mathrm{L}$ & 164 & 0.006 & 5.87 & -6 & -74 & -28 \\
\hline Dorsolateral prefrontal cortex & $\mathrm{R}$ & 108 & 0.043 & 5.54 & 40 & 36 & 18 \\
\hline \multicolumn{8}{|l|}{ House task: high effort $>$ low effort } \\
\hline Anterior insula & $\mathrm{R}$ & 675 & $<0.001$ & 6.69 & 38 & 20 & -2 \\
\hline Dorsal anterior cingulate cortex & $\mathrm{R}$ & 309 & 0.001 & 5.77 & 4 & 22 & 44 \\
\hline Anterior insula & $\mathrm{L}$ & 359 & $<0.001$ & 5.23 & -30 & 26 & -2 \\
\hline Intraparietal sulcus (extending into middle occipital gyrus) & $\mathrm{R}$ & 392 & $<0.001$ & 5.01 & 24 & -62 & 30 \\
\hline Middle occipital gyrus & $\mathrm{L}$ & 202 & 0.002 & 4.56 & -30 & -88 & 22 \\
\hline \multicolumn{8}{|l|}{ Conjunction (high effort $>$ low effort in face task and house task) } \\
\hline Anterior insula & $\mathrm{R}$ & 526 & $<0.001$ & 6.11 & 36 & 20 & -4 \\
\hline Anterior insula & $\mathrm{L}$ & 306 & $<0.001$ & 5.52 & -34 & 20 & -4 \\
\hline Dorsal anterior cingulate cortex & $\mathrm{R}$ & 276 & 0.001 & 5.04 & 6 & 24 & 40 \\
\hline Intraparietal sulcus & $\mathrm{R}$ & 247 & 0.002 & 4.97 & 24 & -62 & 30 \\
\hline
\end{tabular}

L, Left; R, right.

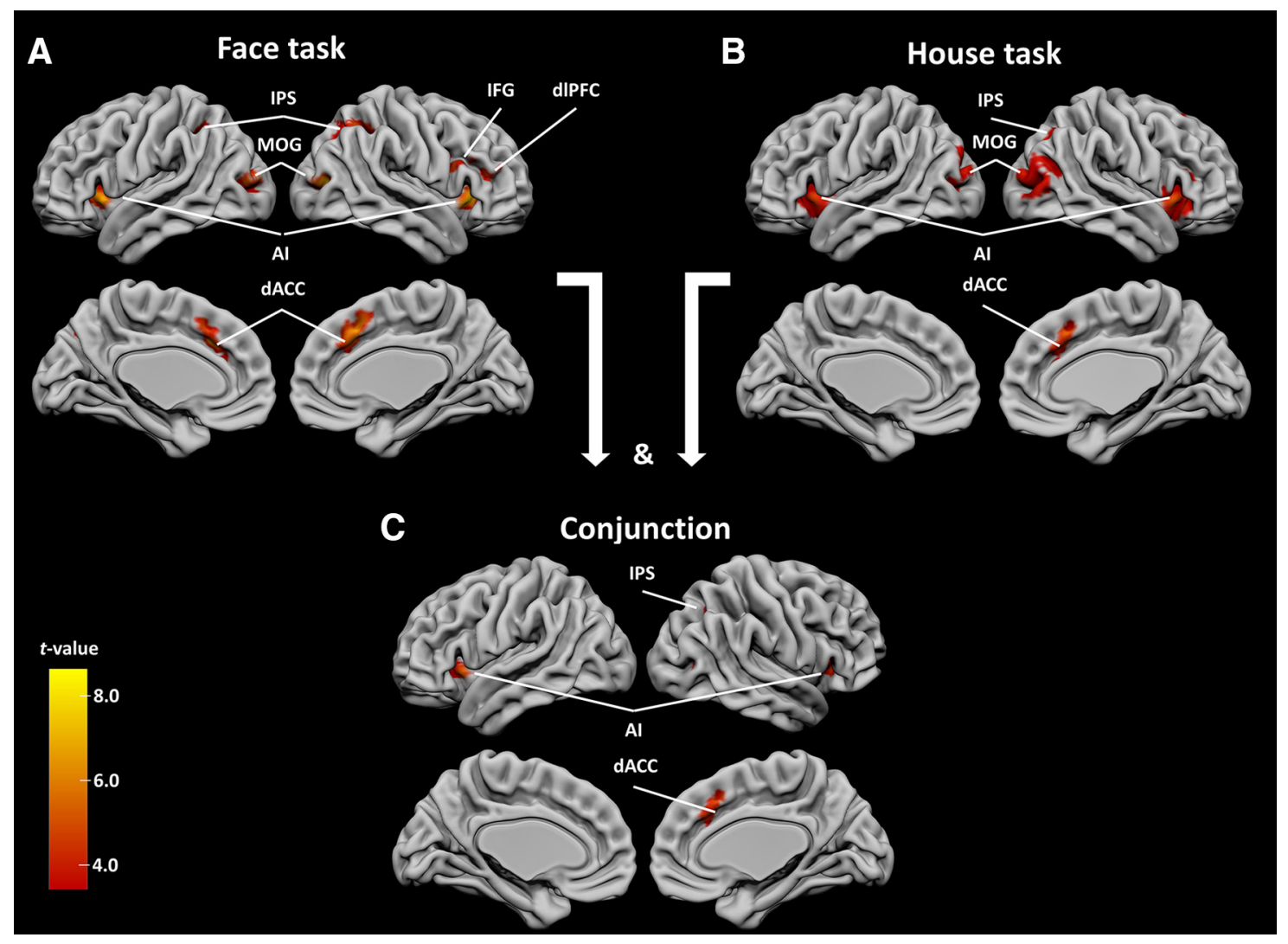

Figure 4. $\quad \boldsymbol{A}, \boldsymbol{B}$, Brain activation in the high-effort versus low-effort demand condition on the face task $(\boldsymbol{A})$ and the house task $(\boldsymbol{B})$. $\boldsymbol{C}$, Conjunction analysis showing mutual activation for the high-effort versus low-effort demand condition on both the face and house tasks.

\section{Eye movements}

Analysis of the eye movement data revealed no main effect of Task $\left(F_{(1,23)}=0.05 ; p=0.83 ; \eta^{2}=<0.001\right)$, Effort $\left(F_{(1,23)}=2.01\right.$; $\left.p=0.17 ; \eta^{2}=0.005\right)$, or an interaction between Task and Effort
$\left(F_{(1,23)}<0.01 ; p=0.98 ; \eta^{2}<0.001\right)$. These findings were confirmed through the computation of Bayes factors $\left(\mathrm{BF}_{01}\right)$, which showed evidence in favor of the null hypothesis and not in favor of a Task effect $\left(\mathrm{BF}_{01}=4.71\right)$ or of an Effort effect $\left(\mathrm{BF}_{01}=1.38\right)$. 
Table 3. Summary of the activation clusters from the gPPI analysis

\begin{tabular}{|c|c|c|c|c|c|c|c|}
\hline \multirow[b]{3}{*}{ Region } & \multirow[b]{3}{*}{ Side } & & & \multicolumn{4}{|c|}{$\begin{array}{l}\text { Peak-level } \\
\text { statistics }\end{array}$} \\
\hline & & \multicolumn{2}{|c|}{$\begin{array}{l}\text { Cluster-level } \\
\text { statistics }\end{array}$} & \multirow[b]{2}{*}{$\begin{array}{l}t \\
\text { value }\end{array}$} & \multicolumn{3}{|c|}{$\begin{array}{l}\text { MNI } \\
\text { coordinates }\end{array}$} \\
\hline & & $\begin{array}{l}\text { Cluster } \\
\text { size }\end{array}$ & $p$ (FWE) & & $x$ & $y$ & $z$ \\
\hline \multicolumn{8}{|l|}{ Face task } \\
\hline Occipital pole & $\mathrm{L}$ & 670 & $<0.001$ & 6.67 & -12 & -94 & -12 \\
\hline Fusiform face area & L & 89 & 0.026 & 5.72 & -42 & -52 & -20 \\
\hline Fusiform face area & R & 222 & $<0.001$ & 5.67 & 36 & -48 & -20 \\
\hline Occipital pole & $\mathrm{R}$ & 111 & 0.008 & 4.64 & 18 & -84 & 12 \\
\hline \multicolumn{8}{|l|}{ House task } \\
\hline $\begin{array}{l}\text { Temporo-occipital, } \\
\text { including PPA and } \\
\text { FFA }\end{array}$ & R & 1425 & $<0.001$ & 6.36 & 36 & -86 & 8 \\
\hline Lateral occipital superior & L & 881 & $<0.001$ & 5.97 & -28 & -78 & 22 \\
\hline Precentral gyrus & $L / R$ & 116 & 0.006 & 5.02 & 0 & -8 & 66 \\
\hline SMA & $\mathrm{L}$ & 191 & $<0.001$ & 4.92 & -32 & -12 & 48 \\
\hline Lateral occipital inferior & L & 79 & 0.045 & 3.86 & -46 & -74 & -8 \\
\hline
\end{tabular}

L, Left; R, right.

There was also more evidence for the null model compared with a model with both Task and Effort $\left(\mathrm{BF}_{01}=6.49\right)$, and compared with a full model that included both effects and their interaction $\left(\mathrm{BF}_{01}=32.0\right)$. On average, participants fixated on $33.2 \%$ of the trials, meaning they generally adhered poorly to the instruction to refrain from eye movements but did so in every condition equally.

\section{Discussion}

Effort investment is thought to be implemented in a hierarchical manner with a crucial role for dACC at the top of this hierarchy (Holroyd and Yeung, 2012; Shenhav et al., 2013; Verguts et al., 2015). In this study, we investigated how this effort investment is implemented in the brain. We used a face detection task and a house detection task with different effort levels, and showed that increased effort investment is reflected in a general increased activation of dACC and related areas, independent of the task at hand. Importantly, we also showed an effort-induced strengthening of connectivity between dACC and specialized lower-level perceptual areas, depending on the performed task. The increased functional connectivity between dACC and lower-level areas emerged in the high-effort condition, where effort investment was rated higher. This connectivity was also task specific: stronger dACC-FFA connectivity was found when high-effort versus low-effort faces had to be detected, while stronger dACCPPA connectivity was found when high-effort versus low-effort houses had to be detected. This fits with the proposed hierarchical position of dACC, allocating resources to task-relevant areas.

One interpretation of the connectivity findings is that dACC amplifies the signal in task-relevant areas to increase performance in difficult conditions (i.e., effort-production account). In the present study, the signal-to-noise ratio of images in the higheffort conditions was low, meaning that they lacked strength to elicit robust bottom-up activation of FFA or PPA. Such activation is needed to make accurate decisions on the content of the presented image (Heekeren et al., 2004; Lamichhane and Dhamala, 2015; Tremel and Wheeler, 2015). The increased connectivity between dACC and perceptual areas may serve as a compensatory mechanism for the lack of clear perceptual

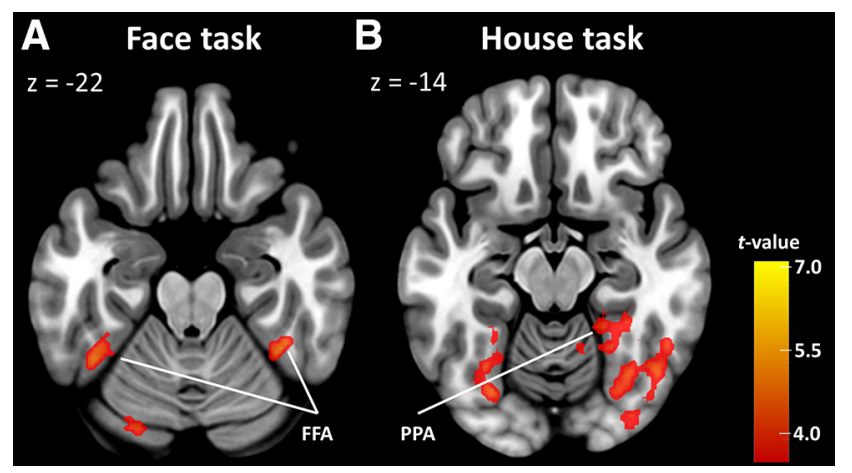

Figure 5. Whole-brain connectivity with $\operatorname{dACC} . \boldsymbol{A}, \boldsymbol{B}$, The connectivity values are contrasted between high-effort and low-effort demands in the face task $(\boldsymbol{A})$ and the house task (B).

evidence present in the stimulus. Since participants knew beforehand what type of stimulus they had to detect, increased input from dACC may function to optimize neural processing of the stimulus by specialized areas (FFA or PPA).

The exact neural mechanism underlying this optimization may be explained by response sensitization of task-relevant areas. There are several ways this might be implemented. For example, it has been proposed that stimulus-induced dynamics in cortical areas can be augmented by increasing their background activity (Chawla et al., 1999; Kastner et al., 1999; Beck and Kastner, 2009). This may result in an increased synchronization of the neurons representing a stimulus (Fries et al., 2001; Buschman and Kastner, 2015). In addition, top-down influences may also decrease noise correlations between neurons in a region. Decreased noise correlations lead to an increase of the signal-tonoise ratio and hence the amount of information encoded by the neuronal ensemble (Gilbert and Li, 2013; Ramalingam et al., 2013). Yet another explanation may be a top-down-induced increase in neural gain in task-relevant regions (Aston-Jones and Cohen, 2005). Increasing gain suppresses weak activation (typically, noise) and increases strong activation (typically, signal), thus functionally increasing the signal-to-noise ratio. Processes like these can give a cortical area an advantage in subsequent stimulus processing.

On a neural level, cognitive effort production may thus be seen as an attempt to overcome a compromised signal in a population of neurons. This idea can also be applied to neuronal fatigue, where adaptation may lead to a reduced signal-to-noise ratio in a brain area, as a result of repeatedly performing a cognitive action. To compensate for this neuronal fatigue, effort may be implemented as a stronger control signal that establishes the necessary signal-to-noise ratio in the relevant neural population (Müller and Apps, 2019). If this is correct, then one would expect stronger connectivity between effort-producing areas (dACC) and task-specific regions toward the end of the task. However, the current results lend no support for such a mechanism, as exploratory brain connectivity analyses showed no effects of timeon-task. Response sensitization of task-relevant areas is also in accordance with the proposed role of dACC in specification of the effort allocation signal. From this perspective, dACC would be involved in the decision about which control signal to apply (i.e., whether to focus on faces or houses) and with what level of intensity (Shenhav et al., 2013). This explanation is consistent with increased connectivity with FFA and PPA, initiated by dACC. 
A

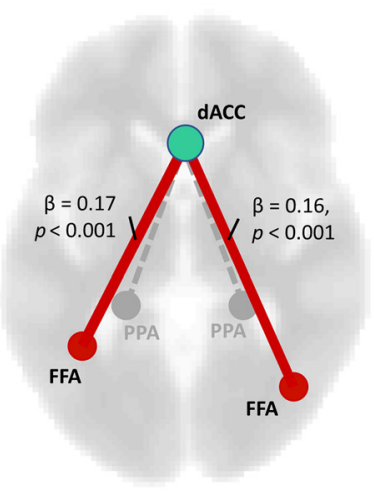
t-value
-4.63

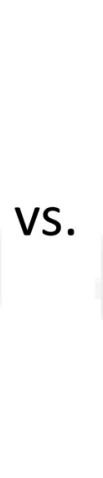

B

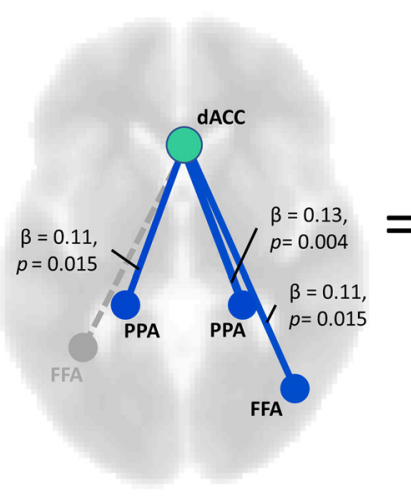

C Face vs. House task

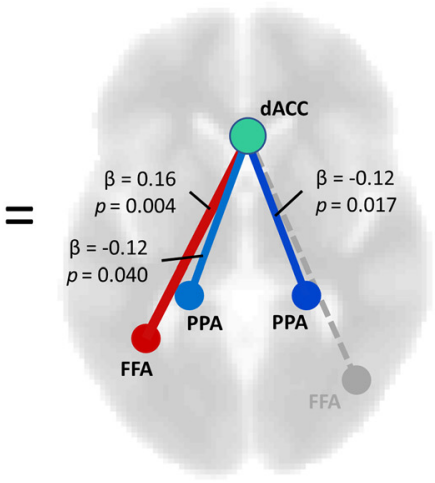

Figure 6. ROl-to-ROl connectivity results with $\mathrm{AACC}$ as seed, and bilateral FFA and PPA as target regions. $\boldsymbol{A}, \boldsymbol{B}$, Effort-induced connectivity on the face task $(\boldsymbol{A})$ and the house task $(\boldsymbol{B})$. $\boldsymbol{C}$, Difference between $\boldsymbol{A}$ and $\boldsymbol{B}$ : difference in effort-increased connectivity between the face task and house task. Red, Increased connectivity on face task; blue, increased activity on house task. Width of the lines corresponds to the strength of the effects.

A second interpretation of the connectivity findings is also possible (effort requirement account). It has been suggested that dACC monitors the current circumstances and tracks how well the cognitive system is meeting task demands (Botvinick et al., 2004; Shenhav et al., 2013). In the current experiment, this would imply that dACC may be informed by task-related areas about the greater effort required to solve the task. For example, the smaller perceptual difference between target-present and targetabsent trials in the high-effort condition may have triggered response conflict. This conflict may have served as the indicator to allocate additional cognitive effort, reflected in conflictinduced activation of dACC in the high-effort condition (Botvinick et al., 2001; Braver, 2012). This explanation would be compatible with an increased connectivity in the opposite direction: from task-relevant areas to dACC.

In the effort requirement account, dACC tracks the adequacy of the implemented effort. When performance deteriorates, for example because of inadequate filtering of distraction or conflict, an increased need for effort will be signaled. This mechanism can be extended to conditions where dACC monitors and specifies behavior to maximize value (Shenhav et al., 2017). In that case, dACC integrates effort costs and reward values and is involved in the decision whether or not it is worthwhile to invest a given level of effort (Klein-Flugge et al., 2016; Chong et al., 2017). This role of dACC in effort-based decision-making is supported by the finding that inactivation of dACC in rats reduced the willingness to invest effort, yet did not affect performance on cognitively demanding tasks (Hosking et al., 2014). In the present study, however, no reward was offered and no cost-benefit decision had to made. Still, increased dACC activity was found, indicating that $\mathrm{dACC}$ also operates whenever more effort is required, regardless of cost-benefit decision-making (but see Vassena et al., 2014; Engström et al., 2015).

The effort production and effort requirement accounts are not mutually exclusive and are in fact proposed to be integrated by dACC (Shenhav et al., 2013). They are also both compatible with the present results, given that connectivity analyses cannot attest to directionality.

We also found that activity in dACC scaled with the level of effort investment, independent of the task. In the high-effort conditions, larger dACC activity was found than in the low-effort conditions. Conjunction analysis showed that this was true for both the face-detection and house-detection task, with jointly activated AACC, bilateral AI, and right IPS. These areas thus constitute a task-independent network of brain regions involved in effortful behavior. Within this network, the importance of dACC for effortful behavior converges with model simulations showing that dACC-lesioned rats are less likely to engage in effortful behavior (Holroyd and Mcclure, 2015), and by the fact that dACC lesions are associated with a lack of motivation or anergia (Cohen et al., 1999; Walton et al., 2002; Holroyd and Yeung, 2012). It is also consistent with studies relating dACC activity to self-reported effort investment (Mulert et al., 2005), will to persevere (Parvizi et al., 2013), and anticipation of effortful tasks (Croxson et al., 2009; Prevost et al., 2010; Kurniawan et al., 2013). However, the exact way in which dACC is involved in effort-based decision-making, effort production, or effort requirement is a topic that warrants future consideration.

Another function often ascribed to dACC is the monitoring of errors (Gehring et al., 1993; Bush et al., 2000). In the current study, however, no error-related effects on brain activity or connectivity were found. Indeed, the current paradigm offered not much opportunity for error monitoring for two reasons. First, participants did not receive trial feedback. Second, it is hard to become metacognitively aware of an error in the high-effort condition because there is never clear evidence in favor of one of the two response options. In addition, participants had to withhold their responses for $1 \mathrm{~s}$. This is different from speeded response tasks where participants occasionally slip and make an error while the correct response is readily available. In such cases, participants often do become aware of the error they made, even without feedback.

Together with dACC activity, we also observed effort-induced activity in bilateral AI and right IPS on both tasks. These regions are often conjointly activated, together with dlPFC, on a wide range of cognitive tasks that demand attention, working memory, or cognitive control (Corbetta and Shulman, 2002; Dosenbach et al., 2006; Menon and Uddin, 2010; Nelson et al., 2010). Anterior insula has traditionally been related to the detection of salient events (Downar et al., 2002; Menon and Uddin, 2010) and attentional control (Nelson et al., 2010). In the present study, saliency of the presented stimuli was constant. The function of AI therefore seems more consistent with accounts postulating that AI subserves maintenance of a task-set (Dosenbach et al., 2006) or tonic 
alertness (Sadaghiani and D'Esposito, 2015). Such processes may have been more profound in the high-effort conditions. For example, a (tonic) activation of AI in high-effort conditions may alert the system that the demand to detect a target is higher (Han et al., 2019). This way, dACC may also be informed to intensify its control signal to task-relevant areas.

In conclusion, we showed increased effort-induced connectivity between dACC and lower-level perceptual areas (FFA or PPA), specific to the performed task. dACC was part of a broader network consisting also of AI and IPS that is more strongly activated when effort demands are high, independent of the task at hand. We conclude that AACC, AI, and IPS constitute a general effort-responsive network and that the neural implementation of cognitive effort may involve dACC-initiated response sensitization of task-dependent areas.

\section{References}

Apps MAJ, Grima LL, Manohar S, Husain M (2015) The role of cognitive effort in subjective reward devaluation and risky decision-making. Sci Rep 5:16880.

Aston-Jones G, Cohen JD (2005) An integrative theory of locus coeruleusnorepinephrine function: adaptive gain and optimal performance. Annu Rev Neurosci 28:403-450.

Beck DM, Kastner S (2009) Top-down and bottom-up mechanisms in biasing competition in the human brain. Vision Res 49:1154-1165.

Behzadi Y, Restom K, Liau J, Liu TT (2007) A component based noise correction method (CompCor) for BOLD and perfusion based fMRI. Neuroimage 37:90-101.

Boksem MAS, Tops M (2008) Mental fatigue: costs and benefits. Brain Res Rev 59:125-139.

Botvinick MM, Braver TS, Barch DM, Carter CS, Cohen JD (2001) Conflict monitoring and cognitive control. Psychol Rev 108:624-652.

Botvinick MM, Cohen JD, Carter CS (2004) Conflict monitoring and anterior cingulate cortex: an update. Trends Cogn Sci 8:539-546.

Botvinick MM, Huffstetler S, McGuire JT (2009) Effort discounting in human nucleus accumbens. Cogn Affect Behav Neurosci 9:16-27.

Braver TS (2012) The variable nature of cognitive control: a dual mechanisms framework. Trends Cogn Sci 16:106-113.

Buschman TJ, Kastner S (2015) From behavior to neural dynamics: an integrated theory of attention. Neuron 88:127-144.

Bush G, Luu P, Posner MI (2000) Cognitive and emotional influences in anterior cingulate cortex. Trends Cogn Sci 4:215-222.

Chawla D, Rees G, Friston KJ (1999) The physiological basis of attentional modulation in extrastriate visual areas. Nat Neurosci 2:671-676.

Chong TTJ, Apps M, Giehl K, Sillence A, Grima LL, Husain M (2017) Neurocomputational mechanisms underlying subjective valuation of effort costs. PLoS Biol 15:e1002598.

Cohen RA, Kaplan RF, Zuffante P, Moser DJ, Jenkins MA, Salloway S, Wilkinson H (1999) Alteration of intention and self-initiated action associated with bilateral anterior cingulotomy. J Neuropsychiatry Clin Neurosci 11:444-453.

Corbetta M, Shulman GL (2002) Control of goal-directed and stimulusdriven attention in the brain. Nat Rev Neurosci 3:201-215.

Crottaz-Herbette S, Menon V (2006) Where and when the anterior cingulate cortex modulates attentional response: combined fMRI and ERP evidence. J Cogn Neurosci 18:766-780.

Croxson PL, Walton ME, Reilly JXO, Behrens TEJ, Rushworth MFS (2009) Effort-based cost-benefit valuation and the human brain. J Neurosci 29:4531-4541.

Danielmeier C, Eichele T, Forstmann BU, Tittgemeyer M, Ullsperger M (2011) Posterior medial frontal cortex activity predicts post-error adaptations in task-related visual and motor areas. J Neurosci 31:1780-1789.

Di X, Biswal BB (2017) Psychophysiological interactions in a visual checkerboard task: reproducibility, reliability, and the effects of deconvolution. Front Neurosci 11:1-16.

Dosenbach NUF, Visscher KM, Palmer ED, Miezin FM, Wenger KK, Kang HC, Burgund ED, Grimes AL, Schlaggar BL, Petersen SE (2006) A core system for the implementation of task sets. Neuron 50:799-812.
Dosenbach NUF, Fair DA, Cohen AL, Schlaggar BL, Petersen SE (2008) A dual-networks architecture of top-down control. Trends Cogn Sci 12:99105.

Downar J, Crawley AP, Mikulis DJ, Davis KD (2002) A cortical network sensitive to stimulus salience in a neutral behavioral context across multiple sensory modalities. J Neurophysiol 87:615-620.

Engström M, Karlsson T, Landtblom A-M, Craig AD (Bud) (2015) Evidence of conjoint activation of the anterior insular and cingulate cortices during effortful tasks. Front Hum Neurosci 8:1-12.

Esterman M, Yantis S (2010) Perceptual expectation evokes category-selective cortical activity. Cereb Cortex 20:1245-1253.

Fries P, Reynolds JH, Rorie AE, Desimone R (2001) Modulation of oscillatory neuronal synchronization by selective visual attention. Science 291:15601563.

Gehring WJ, Goss B, Coles MGH, Meyer DE, Donchin E (1993) A neural system for error detection and compensation. Psychol Sci 4:385-390.

Gilbert CD, Li W (2013) Top-down influences on visual processing. Nat Rev Neurosci 14:350-363.

Gonzalez-Castillo J, Hoy CW, Handwerker DA, Robinson ME, Buchanan LC, Saad ZS, Bandettini PA (2015) Tracking ongoing cognition in individuals using brief, whole-brain functional connectivity patterns. Proc Natl Acad Sci U S A 112:8762-8767.

Han SW, Eaton HP, Marois R (2019) Functional fractionation of the cinguloopercular network: alerting insula and updating cingulate. Cereb Cortex 29:2624-2638.

Hautus MJ (1995) Corrections for extreme proportions and their biasing effects on estimated values of $\mathrm{d}^{\prime}$. Behav Res Methods Instrum Comput 27:46-51.

Heekeren HR, Marrett S, Bandettini PA, Ungerleider LG (2004) A general mechanism for perceptual decision-making in the human brain. Nature 431:859-862.

Holroyd CB, Mcclure SM (2015) Hierarchical control over effortful behavior by rodent medial frontal cortex: a computational model. Psychol Rev 122:54-83.

Holroyd CB, Yeung N (2012) Motivation of extended behaviors by anterior cingulate cortex. Trends Cogn Sci 16:122-128.

Hosking JG, Cocker PJ, Winstanley CA (2014) Dissociable contributions of anterior cingulate cortex and basolateral amygdala on a rodent cost/benefit decision-making task of cognitive effort. Neuropsychopharmacology 39:1558-1567.

Inzlicht M, Shenhav A, Olivola CY (2018) The effort paradox: effort is both costly and valued. Trends Cogn Sci 22:337-349.

Kanwisher N, McDermott J, Chun MM (1997) The fusiform face area: a module in human extrastriate cortex specialized for face perception. J Neurosci 17:4302-4311.

Kastner S, Pinsk MA, De Weerd P, Desimone R, Ungerleider LG (1999) Increased activity in human visual cortex during directed attention in the absence of visual stimulation. Neuron 22:751-761.

Klein-Flugge MC, Kennerley SW, Friston K, Bestmann S (2016) Neural signatures of value comparison in human cingulate cortex during decisions requiring an effort-reward trade-off. J Neurosci 36:10002-10015.

Kool W, McGuire JT, Rosen ZB, Botvinick MM (2010) Decision making and the avoidance of cognitive demand. J Exp Psychol Gen 139:665-682.

Kurniawan IT, Guitart-Masip M, Dayan P, Dolan RJ (2013) Effort and valuation in the brain: the effects of anticipation and execution. J Neurosci 33:6160-6169.

Kurzban R, Duckworth A, Kable JW, Myers J (2013) An opportunity cost model of subjective effort and task performance. Behav Brain Sci 36:661679.

Lamichhane B, Dhamala M (2015) Perceptual decision-making difficulty modulates feedforward effective connectivity to the dorsolateral prefrontal cortex. Front Hum Neurosci 9:1-9.

Medford N, Critchley HD (2010) Conjoint activity of anterior insular and anterior cingulate cortex: awareness and response. Brain Struct Funct 214:535-549.

Menon V, Uddin LQ (2010) Saliency, switching, attention and control: a network model of insula function. Brain Struct Funct 214:655-667.

Mulert C, Menzinger E, Leicht G, Pogarell O, Hegerl U (2005) Evidence for a close relationship between conscious effort and anterior cingulate cortex activity. Int J Psychophysiol 56:65-80. 
Müller T, Apps MAJ (2019) Motivational fatigue: a neurocognitive framework for the impact of effortful exertion on subsequent motivation. Neuropsychologia 123:141-151.

Nelson SM, Dosenbach NUF, Cohen AL, Wheeler ME, Schlaggar BL, Petersen SE (2010) Role of the anterior insula in task-level control and focal attention. Brain Struct Funct 214:669-680.

Nichols T, Brett M, Andersson J, Wager T, Poline JB (2005) Valid conjunction inference with the minimum statistic. Neuroimage 25:653-660.

O’Reilly JX, Woolrich MW, Behrens TEJ, Smith SM, Johansen-berg H (2012) Tools of the trade: psychophysiological interactions and functional connectivity. Soc Cogn Affect Neurosci 7:604-609.

Parvizi J, Rangarajan V, Shirer WR, Desai N, Greicius MD (2013) The will to persevere induced by electrical stimulation of the human cingulate gyrus. Neuron 80:1359-1367.

Peirce JW (2007) PsychoPy-Psychophysics software in Python. J Neurosci Methods 162:8-13.

Petersen SE, Dubis JW (2012) The mixed block/event-related design. Neuroimage 62:1177-1184.

Prevost C, Pessiglione M, Metereau E, Clery-Melin M-L, Dreher J-C (2010) Separate valuation subsystems for delay and effort decision costs. J Neurosci 30:14080-14090.

Ramalingam N, McManus JNJ, Li W, Gilbert CD (2013) Top-down modulation of lateral interactions in visual cortex. J Neurosci 33:1773-1789.

Sadaghiani S, D'Esposito M (2015) Functional characterization of the cingulo-opercular network in the maintenance of tonic alertness. Cereb Cortex 25:2763-2773.

Schiffer A-M, Muller T, Yeung N, Waszak F (2014) Reward activates stimulus-specific and task-dependent representations in visual association cortices. J Neurosci 34:15610-15620.

Shenhav A, Botvinick MM, Cohen JD (2013) The expected value of control: an integrative theory of anterior cingulate cortex function. Neuron 79:217-240
Shenhav A, Musslick S, Lieder F, Kool W, Griffiths TL, Cohen JD, Botvinick MM (2017) Toward a rational and mechanistic account of mental effort. Annu Rev Neurosci 40:99-124.

Summerfield C, Egner T, Mangels J, Hirsch J (2006) Mistaking a house for a face: neural correlates of misperception in healthy humans. Cereb Cortex 16:500-508.

Tremel JJ, Wheeler ME (2015) Content-specific evidence accumulation in inferior temporal cortex during perceptual decision-making. Neuroimage 109:35-49.

Vassena E, Silvetti M, Boehler CN, Achten E, Fias W, Verguts T (2014) Overlapping neural systems represent cognitive effort and reward anticipation. PLoS One 9:e91008.

Verguts T, Vassena E, Silvetti M (2015) Adaptive effort investment in cognitive and physical tasks: a neurocomputational model. Front Behav Neurosci 9:57.

Visscher KM, Miezin FM, Kelly JE, Buckner RL, Donaldson DI, McAvoy MP, Bhalodia VM, Petersen SE (2003) Mixed blocked/event-related designs separate transient and sustained activity in fMRI. Neuroimage 19:1694-1708.

Walton ME, Bannerman DM, Rushworth MFS (2002) The role of rat medial frontal cortex in effort-based decision making. J Neurosci 22:1099611003

Whitfield-Gabrieli S, Nieto-Castanon A (2012) Conn: a functional connectivity toolbox for correlated and anticorrelated brain networks. Brain Connect 2:125-141.

Willems RM, Peelen MV, Hagoort P (2010) Cerebral lateralization of faceselective and body-selective visual areas depends on handedness. Cereb Cortex 20:1719-1725.

Willenbockel V, Sadr J, Fiset D, Horne GO, Gosselin F, Tanaka JW (2010) Controlling low-level image properties: the SHINE toolbox. Behav Res Methods 42:671-684.

Yarkoni T, Poldrack RA, Nichols TE, Van Essen DC, Wager TD (2011) Large-scale automated synthesis of human functional neuroimaging data. Nat Methods 8:665-670. 